\title{
CAMBIOS ESTRUCTURALES Y TECNOLÓGICOS DE LA GANADERÍA DEL CENTRO SUR DE CORRIENTES, ARGENTINA, EN EL PERÍODO 2005-2015
}

\section{STRUCTURAL AND TECHNOLOGICAL CHANGES IN CATTLE PRODUCTION IN CENTRAL-SOUTH CORRIENTES, ARGENTINA, BETWEEN 2005 AND 2015.}

\author{
Mariana Calvi ${ }^{1 *}$, Hernán Alejandro Urcola ${ }^{2}$, Silvina Cabrini ${ }^{3}$, María Daniela Chavez ${ }^{4}$ \\ ${ }^{1}$ Instituto Nacional de Tecnología Agropecuaria (INTA), Estación Experimental Agropecuaria (EEA) \\ Mercedes, Juan Pujol al Este s/n (3470), Mercedes, Corrientes, Argentina. \\ ${ }^{2}$ INTA EEA Balcarce, Ruta 226 Km 73,5 (7620), Balcarce, Buenos Aires, Argentina. \\ ${ }^{3}$ INTA EEA Pergamino y Universidad Nacional del Noroeste de la Provincia de Buenos Aires \\ (UNNOBA), Av. Frondizi (Ruta 32) Km 4,5 (2700), Pergamino, Buenos Aires, Argentina. \\ ${ }^{4}$ INTA EEA Salta, Ruta Nac. 68 Km 172 (4404), Cerrillos, Salta, Argentina. \\ *Autor para correspondencia E-mail: calvi.mariana@inta.gob.ar
}

\section{RESUMEN}

La ganadería constituye una de las producciones principales de la provincia de Corrientes, Argentina. Por lo tanto, comprender sus cambios es esencial para formular e implementar planes de desarrollo más eficaces. El objetivo de este trabajo fue cuantificar y analizar los cambios en la estructura, la tecnología y la productividad de los sistemas ganaderos de Corrientes, durante el período 2005-2015. En estos años, se recopiló información sobre la superficie, existencias ganaderas y personal empleado, como también datos sobre el manejo del rebaño (rodeo) y la tasa de animales logrados, de 29 sistemas de producción. Se caracterizaron las transformaciones tanto a nivel de la muestra como a nivel de los casos individuales mediante test de medias, test de proporciones y análisis de correlaciones. Posteriormente, se aplicó un análisis de correspondencias múltiples para explorar los cambios en los componentes de la varianza. Finalmente, se realizó un agrupamiento en clústers para caracterizar la diversidad existente al final del período. Se observaron pocos cambios en el promedio de ambas muestras, pero existieron cambios considerables en la estructura y manejo de ciertos establecimientos. Los establecimientos con preponderancia de cría bovina tendieron a intensificar su producción, mientras que los casos con menores dotaciones de mano de obra incrementaron su personal. Los resultados muestran que las explotaciones de mayor escala mejoraron el manejo alimentario y reproductivo del rodeo, lo que se asoció a mayores porcentajes de destete. Al final del período, se diferenciaban dos grupos con distinta escala y tecnología, pero con índices reproductivos y productivos similares.

Palabras clave: sistemas de producción, dinámica, tecnología, ganadería, Corrientes.

\section{ABSTRACT}

Livestock production is one of the main economic activities in Corrientes province, Argentina. Therefore, understanding its dynamics is essential to design and implement efficient development programs. The objective of this study was to quantify the structural, technological and production changes in livestock systems in Corrientes, during the period 2005-2015. In these years, data on 
production area, animal stocks, number of workers, herd management and production parameters were collected from 29 farms. Average and farm-level transformations were characterized through test for mean differences, test of proportions and correlation analysis. Then, a multiple correspondence analysis was used to determine changes in components of variance. Finally, clusters were formed to characterize the diversity of farms existing at the end of the study period. On average, most variables showed slight changes. Nonetheless, substantial changes were detected on individual cases. Farms specialized in cattle tended to intensify their production, while those with lower number of workers tended to increase the amount of labor employed. The results also showed that larger farms improved feeding and reproductive management of the herd, which improved weaning percentages. At the end of the study period, two types of farms with distinct sizes and technologies were identified, but reproductive and productive performances were similar for both groups.

Key words: production systems, dynamics, technology, cattle production, Corrientes.

\section{INTRODUCCIÓN}

La ganadería constituye una de las actividades productivas más importantes del Centro Sur (región que incluye los departamentos de Curuzú Cuatiá, Mercedes, Monte Caseros, Paso de los Libres y Sauce) de la provincia de Corrientes, Argentina. Con más de 2 millones de vacunos, esta región se destaca por su ganadería extensiva sobre pastizales naturales, donde la actividad principal es la cría mixta de bovinos y ovinos, y en menor medida la invernada de vacunos. Según los datos del SENASA (2016), esta región alberga 3.615 establecimientos agropecuarios y 2.014 .742 bovinos, $24 \%$ y $40 \%$ del total provincial, respectivamente. Entre los establecimientos agropecuarios, predominan los que manejan hasta 500 cabezas de ganado. Sin embargo, un $60 \%$ del stock bovino es manejado por establecimientos que tienen entre 500 y 5.000 cabezas de ganado. Es decir, el estrato de establecimientos con entre 500 y 5.000 cabezas es el más significativo en términos de sus aportes al desarrollo regional.

En general, el sector ganadero provincial es visto como poco productivo e innovador, presentando una importante brecha tecnológica con respecto a los rendimientos potenciales. Sin embargo, varios estudios coinciden en la existencia de distintos grupos de productores con distintos comportamientos en lo que a adopción tecnológica se refiere (Calvi, 2009; Velasco Fuenmayor et al., 2009). Calvi (2009) identificó 4 tipos de productores, según su nivel de capitalización y grado de adopción tecnológica, desde familiares de baja adopción hasta empresariales de alta adopción. Por un lado, los sistemas de producción empresarial de alta adopción se caracterizaban por haber incorporado una serie de prácticas de manejo recomendadas para la cría, como el servicio anticipado de las vaquillonas, la suplementación estratégica, el destete precoz y la utilización de un pequeño porcentaje de pasturas. Por otro lado, los sistemas familiares no utilizaban este tipo de técnicas de manejo, sino que empleaban una estrategia productiva que incluía un doble servicio (en primavera y otoño), el mantenimiento de una carga más alta, y una mayor participación de los ovinos en la carga animal total de los establecimientos. Es de destacar, que los sistemas familiares presentaron los mejores resultados productivos y económicos por hectárea, los cuales se explican por las mayores cargas y la diversificación con ovinos. Calvi (2008) también estimó la brecha tecnológica por región y actividad, como la diferencia en porcentaje entre el rendimiento promedio y el rendimiento potencial en $\mathrm{kg}$ de carne $\mathrm{ha}^{-1}$. El análisis arroja diferencias entre estos rendimientos del $51 \%$ en cría pura y $43 \%$ en ciclo completo para el Centro Sur.

La relación entre tamaño y número de establecimientos con su nivel tecnológico y su productividad, y como estas relaciones evolucionan en el tiempo, ha sido de interés continuo para investigadores y diseñadores de políticas de desarrollo sectorial (Gonzalez, 2005; Rada y Fuglie, 2018). En los sistemas de producción ganaderos el tema de la adopción tecnológica requiere de más investigación, tanto su seguimiento en el tiempo como sus variaciones a través de los sistemas de distinta escala $u$ orientación de la producción. Esto es así, ya que hay factores externos del contexto social, técnico y empresario que limitan la adopción (Rearte, 2010; Giancola et al., 2013). Numerosos estudios analizan la evolución tecnológica, productiva y los cambios en el uso del suelo de los sistemas de producción predominantes en Argentina; sin embargo, varios de estos esfuerzos han sido orientados a regiones de producción agrícola de la llanura Pampeana (Paruelo et al., 2005; Bert et al., 2011). Para sistemas ganaderos, en general, y para los de la provincia de Corrientes en particular, no se conocen estudios que caractericen la evolución de los sistemas de producción a lo largo del tiempo. Conocer esta dinámica es importante para la generación de planes de desarrollo 
regional, para el diseño de líneas de crédito específicas y para monitorear la sustentabilidad de la producción.

De acuerdo a los antecedentes presentados, el objetivo del presente trabajo es: cuantificar y analizar los cambios en la estructura, en la tecnología y en el nivel productivo de los sistemas de producción ganaderos de la región Centro Sur de Corrientes, Argentina, durante el período 2005-2015.

\section{MATERIALES Y MÉTODOS}

\section{Recolección de datos}

El presente trabajo analiza las transformaciones experimentadas por los sistemas de producción durante el período 2005-2015, utilizando como momentos de muestreo de datos los años 2005 y 2015. En el año 2005 se tomó una muestra poblacional representativa del tipo aleatoria por estrato y departamento integrada por 43 productores. Durante el período de análisis algunas de las empresas se dividieron, otras se vendieron, de modo que sólo se pudieron volver a entrevistar 29 establecimientos. Para estudiar las transformaciones ocurridas se utilizó el subset de datos correspondiente a los 29 establecimientos agropecuarios para los que se tenían observaciones en los dos momentos. En los años 2005 y 2015, se analizaron establecimientos que tenían entre 500 y 5.000 cabezas de bovinos, del Centro Sur de Corrientes, dada su importancia y representatividad regional.

En ambos momentos de muestreos se realizaron entrevistas semi-estructuradas a cada uno de los productores o administradores de los establecimientos incluidos en la muestra. Las entrevistas relevaban mayoritariamente aspectos estructurales, tecnológicos y productivos, incluyendo información sobre la superficie operada, el stock ovino y bovino del establecimiento, la dotación de personal y la participación de la mano de obra (MO) familiar en las labores generales. Las entrevistas también recolectaban información sobre el manejo sanitario, alimentario y reproductivo del rebaño (rodeo), como así también datos sobre la carga animal y la tasa de destete lograda. Las preguntas contenidas en las entrevistas realizadas se referían a los años 2005 y 2015. A partir de la información relevada, se construyeron variables adicionales que se consideraron relevantes para caracterizar los sistemas productivos, como la dotación de personal por cada 500 equivalente vaca (EV). El $\mathrm{EV}$ es un coeficiente que permite igualar todas las categorías de hacienda que componen el rodeo pudiendo sumarlas. El listado de las variables utilizadas en el análisis se presenta en la Tabla 1.

\section{Análisis estadístico}

En primer lugar, se aplicó la estadística descriptiva para caracterizar ambas sub-

Tabla 1. Nombre y unidad de las variables seleccionadas para el análisis de la ganadería de Corrientes, Argentina.

Table 1. Name and unit for the variables used in the analysis of cattle production of Corrientes, Argentina.

\begin{tabular}{lll}
\hline Grupo & Nombre & Unidad \\
\hline Estructurales & Superficie propia & Hectáreas \\
& Superficie de terceros* & Hectáreas \\
& Superficie ganadera & Hectáreas \\
& Existencia bovina & Cabezas \\
& Dotación de personal cada 500 EV & Personas \\
& Participación de la MO familiar & $\%$ \\
& Participación del engorde bovino & $\%$ \\
& Participación de la carga ovina & $\%$ \\
\hline Tecnológicas & Edad de la vaquillona al primer servicio (entore) & Meses \\
& Duración del servicio bovino & Días \\
& Uso de verdeos bovino & $\%$ \\
& Suplementación energética-proteica bovino * & $\%$ casos \\
& Suplementación mineral bovino * & $\%$ casos \\
\hline Productivas & Carga animal & EV/ha \\
& Destete bovino & $\%$ \\
& Señalada ovina * & $\%$ \\
\hline
\end{tabular}

*Variables no utilizadas en los análisis de correspondencias múltiples y clústers. 
muestras. Se calcularon promedios, medianas, errores estándar y cuartiles para cada variable. Se calcularon las diferencias entre las medias de cada una de las variables, entre ambos momentos de muestreo. Para analizar estadísticamente las diferencias encontradas entre 2005 y 2015, se utilizaron diferentes test según el tipo $\mathrm{y}$ distribución de las variables. Sobre las diferencias, se utilizó la prueba $t$ en los casos en los que la variable tuviera una distribución normal, y el test no paramétrico de Kruskal Wallis en caso contrario (la prueba de normalidad se realizó con el test de Shapiro-Wilks considerando un $\alpha$ $=0,05)$. Para las variables binomiales, se aplicó el test Chi cuadrado para las diferencias de proporciones. De esta forma se caracterizaron las transformaciones ocurridas a nivel de la muestra en su conjunto, mediante el análisis de los cambios en el promedio de las variables consideradas. De forma complementaria, también se estudiaron los cambios experimentados por los casos individuales y su relación con el resto de sus características.

Se analizaron estadísticamente las correlaciones entre los cambios en cada variable (las diferencias entre el valor en 2015 menos el valor en 2005) y los valores iniciales de todas las variables consideradas.

$$
\begin{array}{cc}
\Delta x=\left(x_{2015}-x_{2005}\right) & \text { Ecuación 1 } \\
\operatorname{corr}\left(\Delta x, x_{2005}\right) & \text { Ecuación 2 }
\end{array}
$$

donde $x$ representa la variable de interés.

Las correlaciones definidas en las ecuaciones (1) y (2) informan sobre la relación entre los cambios ocurridos en los aspectos estructurales, de manejo y productivos de cada caso con las características de los mismos al inicio del período de estudio. Correlaciones positivas entre una variable diferencia y otra variable de referencia (la cuál puede ser la misma variable) indican que los mayores incrementos en los cambios de la variable diferencia entre el inicio y el fin del período se asocian a valores altos de la variable de referencia, al inicio del período. Este análisis permite detectar, por ejemplo, si los cambios en el tamaño del stock bovino, se asocian o no a la escala que los establecimientos tenían en 2005.

En segundo término, se aplicó un análisis de correspondencias múltiples (ACM) a las muestras de cada año para explorar los cambios en los componentes de la varianza, durante el período analizado. Este análisis es similar al desarrollado por Ricard et al. (2015). El ACM permite reducir la complejidad multidimensional de los datos a un número menor de variables independientes que explican una porción sustancial de la varianza del set de datos original. Además, este análisis muestra cómo la varianza y la correlación entre las variables relacionadas a la estructura, tecnología y productividad de los establecimientos, cambia a lo largo del tiempo y se redistribuye en el espacio bidimensional comprendido por los dos primeros ejes del análisis.

Finalmente, para caracterizar la diversidad existente al final del período de análisis, se realizó un agrupamiento mediante el análisis clústers (conglomerados) para el año 2015. Partiendo de una muestra de individuos caracterizados por un número dado de variables, el análisis de clústers permite clasificar a estos individuos en grupos de manera tal quelosindividuos de un grupo (clúster) sean tan similares entre sí como sea posible, y que los grupos formados sean tan disimilares entre ellos como sea posible (Greenacre, 2017). La idea subyacente en el análisis de clústers es que los grupos formados tienen distintas características intrínsecas y que pueden recibir un tratamiento distinto al que recibe otro grupo. En el presente análisis se utilizó el método de clúster jerárquico, utilizando distancia euclídea y criterio Ward. Los conglomerados seleccionados, obedecen a los cortes realizados en el dendograma a una distancia igual al 75\% de la distancia máxima.

\section{RESULTADOS Y DISCUSIÓN}

Los valores medios para las variables estructurales, tecnológicas y productivas, al inicio y al final del período analizado y los cambios entre los mismos se presentan en la Tabla 2. En la mayoría de los casos hay pequeñas diferencias entre los momentos de muestreo. Entre las variables de estructura, no se observan cambios estadísticamente significativos, pero si los hay entre las variables tecnológicas. Estos cambios son el incremento en el uso de verdeos (la superficie sembrada con verdeos pasó de $0,20 \%$ a $1,73 \%$ de la superficie operada del campo), la disminución de la duración del servicio de monta (antes el período de servicio era de 185 días y al final era de 170 días), el incremento en la suplementación energético-proteica (al final un $28 \%$ más de establecimientos utilizan la suplementación energético-proteica) y la disminución en la suplementación mineral (al final un 17\% menos de establecimientos suplementan con minerales). Salvo esta última tecnología, las demás apuntan a una mejora en la actividad de cría.

Es esperable que la evolución de estas variables esté relacionada. Una mejor alimentación basada en el uso de verdeos y suplementación energéticoproteica permite un crecimiento más rápido de las vaquillonas de reposición posibilitando un entore a edad más temprana. Por otro lado, el aumento en el uso de suplementación energética, pudo haber estado favorecido por la menor 
Tabla 2. Valores promedio, errores estándar, diferencia y significancia para las variables estructurales, tecnológicas y productivas, al inicio y al final del período de análisis.

Table 2. Mean values, standard errors, differences and p-values for structural, technological and production variables, at the beginning and end of the sampling period.

\begin{tabular}{|c|c|c|c|c|c|}
\hline Variables & & $\begin{array}{l}\text { Promedio } \\
2005\end{array}$ & $\begin{array}{l}\text { Promedio } \\
2015\end{array}$ & $\begin{array}{r}\text { Diferencia } \\
\text { promedio } \\
2015-2005\end{array}$ & Valor-p \\
\hline \multicolumn{6}{|l|}{ Estructurales } \\
\hline Sup. propia & ha & $2503(323)$ & $2502(323)$ & -1 & $0,8337^{\text {a }}$ \\
\hline Sup. terceros & ha & 338 (155) & 48 (116) & -290 & $0,2448^{a}$ \\
\hline Sup. ganadera & ha & $2736(305)$ & $2493(329)$ & -243 & $0,4460^{\text {a }}$ \\
\hline Exist. bovina & $\mathrm{Cab}$ & $1838(191)$ & $1816(242)$ & -22 & $0,8888^{a}$ \\
\hline Personal c/500 EV & Pers & $1,33(0,12)$ & $1,30(0,10)$ & $-0,04$ & $0,8039^{a}$ \\
\hline MO familiar & $\%$ & $11,69(2,63)$ & $11,93(3,16)$ & 0,24 & $0,9369^{a}$ \\
\hline Engorde bovino & $\%$ & $12,24(3,49)$ & $11,46(1,93)$ & $-0,79$ & $0,2587^{a}$ \\
\hline Carga ovina & $\%$ & $6,56(0,67)$ & $7,06(0,99)$ & 0,50 & $0,9845^{a}$ \\
\hline \multicolumn{6}{|l|}{ Tecnológicas } \\
\hline Edad entore & Meses & $23,14(1,06)$ & $22,82(0,86)$ & $-0,32$ & $0,9851^{a}$ \\
\hline Servicio bovino & Días & $185(11)$ & $170(10)$ & -15 & $0,0172^{a * *}$ \\
\hline Uso verdeos & $\%$ & $0,20(0,11)$ & $1,73(0,80)$ & 1,53 & 0,0167 a** \\
\hline Supl. energ-prot & $\%$ casos & $48(9)$ & $76(8)$ & 28 & $0,0355^{b * *}$ \\
\hline Supl. mineral & $\%$ casos & $86(6)$ & $69(9)$ & -17 & $0,1317^{b *}$ \\
\hline \multicolumn{6}{|l|}{ Productivas } \\
\hline Carga animal & EV/ha & $0,72(0,03)$ & $0,76(0,04)$ & 0,04 & $0,3537^{a}$ \\
\hline Destete bovino & $\%$ & $71,45(1,85)$ & $73,61(2,08)$ & 2,16 & $0,2587^{a}$ \\
\hline Señalada ovina & $\%$ & $72,95(3,15)$ & $73,72(2,22)$ & 1,97 & $0,5188^{a}$ \\
\hline
\end{tabular}

Los números entre paréntesis son los errores estándar.

a Variables continuas. Se implementó sobre la variable de diferencia la prueba de normalidad Shapiro-Wilks, si la distribución resultó normal se hizo el test t, caso contrario se hizo el test Kruskal Wallis.

${ }^{\mathrm{b}}$ Variables binomiales. Se implementó el test de diferencias de proporciones.

** $\mathrm{y}$ * Indican p-valores menores a 0,05 y 0,15 , respectivamente. Fueron testeadas todas las variables relevadas, pero por razones de espacio sólo se presenta una selección de ellas.

relación de precios entre el maíz y el novillo hacia el final del período de análisis $(0,901 \mathrm{vs} 0,514 \mathrm{~kg}$ de novillo por kg de maíz en los años 2005 y 2015, respectivamente). Estos cambios sugieren un uso más intensivo y eficiente del capital hacienda.

Estos resultados indican pocos cambios en el promedio de ambas muestras, pero la Fig. 1 muestra considerable dispersión en las distribuciones de las diferencias de ciertas variables. Esta dispersión de las diferencias indica que algunos establecimientos experimentaron cambios importantes en algunas de sus variables de estructura y manejo. En la Fig. 1 los cambios de mayor magnitud están representados por las variables superficie de terceros, duración del servicio de monta en bovinos y participación de los ovinos en la carga animal total. En las secciones siguientes se analizan los cambios a nivel de las explotaciones individuales y su relación con las características generales de las mismas.

\section{Análisis de correlaciones}

El análisis de correlaciones muestra que los establecimientos que incrementaron su superficie propia entre ambos períodos son los que en 2005 tenían una mayor proporción de superficie alquilada (de terceros) y una mayor proporción de engorde. Estos cambios se visualizan por las correlaciones positivas y significativas entre estas variables en la Tabla 3. Este cambio puede deberse a que los establecimientos con mayor superficie alquilada y mayor engorde se vieron favorecidos por su mayor escala de producción y obtuvieron mayores márgenes económicos que los sistemas de cría pura (con ventas de terneros al destete) lo que les permitió incrementar la superficie propia, durante estos años.

El análisis de correlaciones también indica que la disminución de la cantidad de superficie alquilada entre 2005 y 2015, está asociada a mayor superficie alquilada y mayor participación del engorde en la producción bovina en 2005 (Tabla 3). Esta 


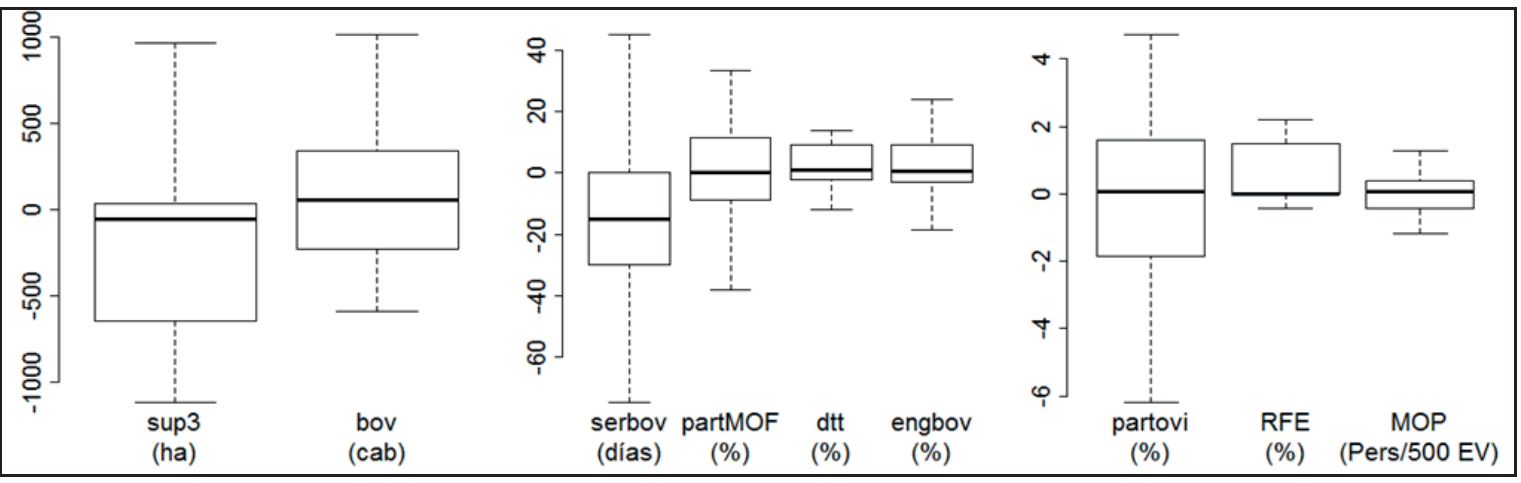

Fig. 1. Diferencias en los valores de variables seleccionadas entre el inicio y el fin del período de muestreo.

Fig. 1. Differences in the values of selected variables between the beginning and end of the sampling period.

Referencias: sup3 es superficie de terceros, bov es existencia bovina, serbov es servicio bovino, partMOF es mano de obra familiar, dtt es destete, engbov es engorde de bovinos, partovi es carga ovina, RFE es uso de verdeos y MOP es personal cada $500 \mathrm{EV}$.

disminución en la superficie alquilada podría deberse al incremento en la relación de precios entre el ternero y el novillo entre inicio y fin del período analizado (precio ternero/precio novillo $=1,18$ en 2005 vs 1,34 en 2015). Estas relaciones indican que en el primer período era necesario un $18 \%$ de la producción para cubrir el costo de compra del ternero, mientras que en 2015 se requería el 34\% para cubrir este costo.

El análisis indica que algunos establecimientos experimentaron cambios diferencialesenel manejo alimentario y reproductivo del rodeo bovino. La Tabla 3 muestra que los establecimientos con mayor edad al primer entore y período de servicio más extenso fueron los que más redujeron estas variables. Al inicio del período, edades de entore mayores y períodos de servicio más extensos se asocian a establecimientos con una menor participación de engorde bovino ${ }^{1}$ (correlaciones de $-0,44(0,06)^{2}$ y de $-0,11(0,66)$, respectivamente), establecimientos con menor uso de verdeos (correlaciones de $-0,27(0,27)$ y $-0,14(0,57)$, respectivamente) y establecimientos con mayor participación de mano de obra familiar (correlaciones de $0,51(0,02)$ y $0,38(0,11)$, respectivamente). Estas características se asocian a explotaciones que desarrollaban una producción más extensiva en el año 2005. Sin embargo, los cambios en las variables entre ambos momentos de muestreo indican que estas explotaciones han implementado cambios tendientes a intensificar la producción.

Por otro lado, el incremento en el uso de verdeos (con diferencia positiva en esta variable), se asocia a los casos con mayores existencias bovinas. Esto se evidencia por la correlación positiva y estadísticamente significativa entre el uso de verdeos y las existencias bovinas (Tabla 3). Además, el incremento en el uso de estos recursos forrajeros se asocia también con una mayor participación del engorde y mayor proporción de superficie alquilada, tal como se observa en las correlaciones positivas de uso de verdeos con respecto a las mencionadas variables (Tabla 3). Estos cambios se deben a que es una práctica común que los establecimientos con mayor número de bovinos arrienden tierra a terceros para realizar el engorde. A su vez, los animales en engorde tienen mayores requerimientos energéticos que el rodeo de cría, lo que justifica un uso proporcionalmente mayor de verdeos.

Con respecto a los cambios en la mano de obra, los resultados indican que los casos que más incrementaron su dotación de mano de obra fueron los que menores dotaciones de este recurso tenían en 2005 (Tabla 3). Este cambio puede deberse a que el $25 \%$ de los establecimientos analizados tenían, en 2005, dotaciones de personal menores a lo recomendado para la zona (1 persona cada $500 \mathrm{EV}$ ).

El análisis de correlación indica que los establecimientos que incrementaron su porcentaje de destetefueron aquellos con mayor participación de ovinos en la carga animal en el inicio del período de análisis (Tabla 3). Muchos autores tiempo atrás han observado la complementación

\footnotetext{
${ }^{1}$ Las correlaciones entre todas las variables de cada uno de los años de muestreo fueron testeadas, pero por razones de espacio se reportan en el texto solo cuando son necesarias para interpretar los cambios ocurridos.

${ }^{2}$ Entre paréntesis se muestran los p-valores asociados a cada coeficiente de correlación.
} 
Tabla 3. Correlación entre el cambio en las variables de interés entre 2005 y 2015 con los valores de las variables en 2005.

Table 3. Correlation of the difference in means between 2005 and 2015 and the means for 2005.

\begin{tabular}{lllcr}
\hline $\begin{array}{l}\text { Diferencia } \\
\text { 2015 - 2005 }\end{array}$ & Valor al inicio (2005) & n & $\begin{array}{c}\text { Correlación de } \\
\text { Pearson }\end{array}$ & Valor-p \\
\hline Sup. Propia & Sup. Terceros & 29 & 0,36 & $0,0573^{*}$ \\
Sup. Propia & Engorde bovino & 29 & 0,31 & $0,1030^{*}$ \\
Sup. Propia & Edad entore & 29 & $-0,29$ & $0,1296^{*}$ \\
Sup. Terceros & Sup. Terceros & 29 & $-0,78$ & $<0,0001^{* *}$ \\
Sup. Terceros & Engorde bovino & 29 & $-0,52$ & $0,0036^{* *}$ \\
Personal c/500 EV & Personal c/500 EV & 29 & $-0,69$ & $<0,0001^{* *}$ \\
Edad entore & Edad entore & 29 & $-0,61$ & $0,0004^{* *}$ \\
Servicio bovino & Servicio bovino & 29 & $-0,35$ & $0,0623^{*}$ \\
Uso verdeos & Sup. Terceros & 29 & 0,35 & $0,0655^{*}$ \\
Uso verdeos & Exist. Bovina & 29 & 0,36 & $0,0527^{*}$ \\
Uso verdeos & Engorde bovino & 29 & 0,56 & $0,0016^{* *}$ \\
Destete bovino & Carga ovina & 16 & 0,61 & $0,0119^{* *}$ \\
\hline
\end{tabular}

** $\mathrm{y}$ * Indican valores $p$ menores a 0,05 y 0,15, respectivamente. Fueron testeadas las correlaciones entre todas las variables presentadas, pero por cuestiones de espacio sólo se reporta algunas que resultaron estadísticamente significativas.

en el pastoreo entre bovinos y ovinos; más tarde Pueyo et al. (1993) determinaron como óptimo una inclusión de ovinos que no supere el $30 \%$ de la carga animal. De esta manera, se aprovecha mejor el pastizal dado las distintas alturas de pastoreo de cada especie animal, a la vez que se promueve un control natural de parásitos (Calvi, 2009; Cetrá et al., 2002; Pereira et al., 2014).

\section{Cambios en los componentes de la varianza}

Esta sección analiza el comportamiento temporal de los componentes de la varianza. La Fig. 2 muestra el comportamiento temporal de las variables discriminantes del ACM. Estas variables se grafican en los ejes correspondientes a los componentes 1 y 2 . La proporción de la varianza explicada por los ejes 1 y 2 es similar para los años 2005 y 2015, 47 y 45\%, respectivamente. Esta similitud en las proporciones de la varianza explicada sugiere que las transformaciones experimentadas por los sistemas pueden ser explicadas con precisión similar al inicio y al final del período de análisis. Para obtener una interpretación más clara de los cambios en la estructura de correlaciones es necesario discriminar entre los factores relacionados a la estructura, la tecnología y la productividad de las explotaciones.

El comportamiento temporal discriminado de los factores de estructura (superficie propia, superficie ganadera, existencia bovina, personal c/500 EV, engorde bovino, carga ovina), manejo (edad entore, servicio bovino, uso verdeos) y productividad (carga animal, destete bovino) son graficados sobre los componentes 1 y 2 (Fig. 2). Con respecto a las variables de estructura, se verifica que la correlación entre la superficie propia y el engorde bovino se incrementó durante el período analizado (-0,05 vs 0,30 , respectivamente). Además, la superficie propia correlaciona mejor con la existencia bovina en 2015, que en 2005 $(0,83$ vs 0,61 , respectivamente). Es decir, los establecimientos con mayor superficie propia incrementaron la participación del engorde y aumentaron su stock de animales, entre ambos períodos. Además, la Fig. 2 muestra que los establecimientos con más stock tienen más carga, en 2015 que en 2005. Por otro lado, la carga animal presenta una correlación mayor con el porcentaje de destete en 2015 que en 2005 (0,35 vs -0,02 con p-valores de 0,10 y 0,92, respectivamente). Es decir, en 2015 los establecimientos con más carga lograban mejores índices de destete, a diferencia de lo que ocurría en 2005. Lo cual se asocia a la mejora en los recursos forrajeros como uso de verdeos y suplementación energético-proteica. El análisis de correlaciones confirma que los mayores incrementos en porcentaje de destete se dieron en los establecimientos que tenían más existencias bovinas en 2005, aunque las correlaciones no son significativas al 10\% (Tabla 3). Estos resultados indican una intensificación diferencial, durante el período de análisis, en el cuál los establecimientos de mayor superficie propia incrementaron su carga animal y esto se asocia a un aumento en el porcentaje de destete.

El análisis de la mano de obra y la productividad de las explotaciones, medida a través del porcentaje de destete, muestra que la cantidad de personal correlaciona mejor con el 


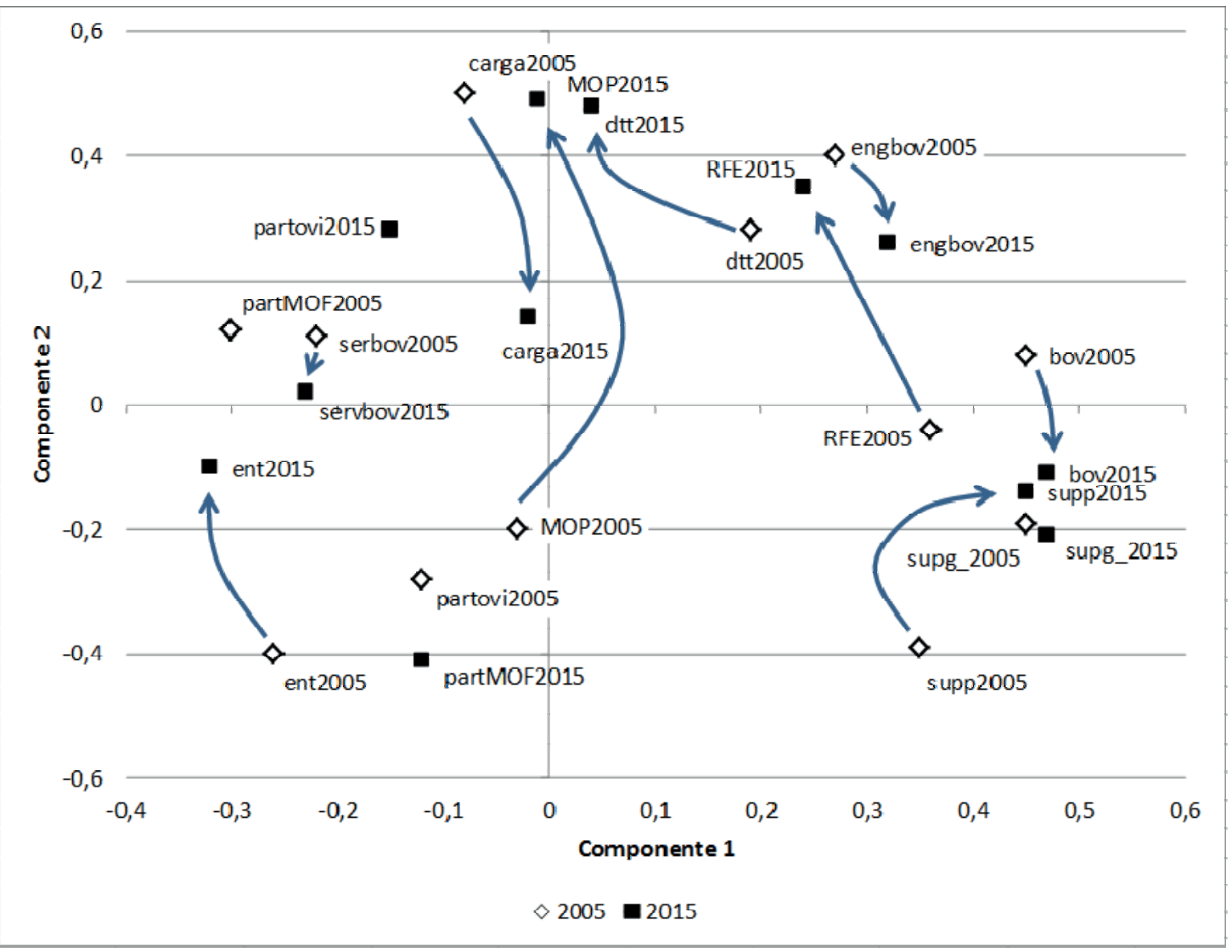

Fig. 2. Componentes de la varianza al principio y al fin del período de análisis para variables de estructura, manejo y productividad de las explotaciones.

Fig. 2. Components of variance at the beginning and end of the study period.

Referencias: supp es superficie propia, supg es superficie ganadera, bov es existencia bovina, serbov es servicio bovino, partMOF es mano de obra familiar, $\mathrm{dtt}$ es destete, engbov es engorde de bovinos, partovi es carga ovina, RFE es uso de verdeos, MOP es personal cada $500 \mathrm{EV}$, carga es carga animal y ent es edad de entore.

porcentaje de destete en 2015 que en 2005 (1,0\% vs $23,0 \%$ ) y sugiere que la mano de obra se ha vuelto más productiva a lo largo del tiempo. Una mayor dotación de mano de obra es necesaria para implementar la suplementación estratégica y un plan sanitario más completo $\mathrm{y}$, por otro lado, puede brindar mayor atención en los momentos críticos del rodeo como parición y destete, lo que redunda en mejores índices productivos.

El comportamiento temporal de las variables relacionadas a la tecnología empleada muestra cambios en el manejo de la alimentación y la reproducción de los rodeos. El uso de verdeos mostró un incremento significativo $(\mathrm{p}<0,05)$ durante el período de análisis (Tabla 2) y la correlación entre el uso de verdeos con la edad de entore y la duración del servicio bovino se volvió más negativa entre años. Esta evolución es esperable ya que una mejor alimentación permite entorar a las vaquillonas a edad más temprana.

Además, la correlación entre el uso de verdeos y el porcentaje de destete se incrementó entre ambos momentos analizados, volviéndose estadísticamente significativa en el año 2015 (coeficientes de correlación de 0,26 y 0,39 y p-valores de 0,27 y 0,06 para el inicio y fin del período de análisis, respectivamente). Este cambio en el comportamiento temporal indica que el uso de verdeos se asocia más fuertemente al número de terneros destetados, hacia el final del período de análisis. Finalmente, tanto para 2005 como para 2015 se verifica que la edad de entore y el tiempo de servicio correlacionan negativamente con las variables de escala superficie ganadera y existencias bovinas, indicando que los establecimientos más grandes tienen un tiempo de servicio menor y realizan el primer entore a menor edad. Este comportamiento sugiere un diferencial tecnológico favorable a las explotaciones de mayor escala. En líneas generales, los resultados muestran una leve tendencia a la intensificación productiva, liderada por las explotaciones de mayor tamaño. 
Análisis de clústers

De acuerdo a las variables seleccionadas se establecieron dos tipos de productores dentro de la población estudiada en el año 2015. Detalles de los valores medios de variables estructurales, tecnológicas y productivas para cada grupo son presentados en la Tabla 4.

El clúster 1, integrado por el $76 \%$ de los productores de la muestra, incluye los establecimientos de menor tamaño, tanto en tierra como en hacienda. El productor posee en propiedad casi la totalidad de la superficie operada, pero en este clúster existen más casos que ceden tierras en alquiler que los que las toman, y por lo tanto la superficie propia de todo el grupo es levemente superior a la superficie ganadera. En estos establecimientos, dedicados principalmente a la cría bovina con un bajo porcentaje de engorde, un $14 \%$ de mano de obra total es aportada por la familia y la cantidad total de mano de obra resulta apropiada para la cantidad de animales disponibles. Los resultados productivos son buenos con respecto al promedio de la zona, alcanzando porcentajes de destete del $73 \%$ en promedio. Además, los productores de este grupo logran mayor porcentaje de señalada en ovinos que el grupo 2 (77\% vs 64\%, respectivamente). En relación a las tecnologías aplicadas, el promedio de este grupo entora a los 2 años de edad, pone en servicio a los vientres durante 6 meses y el $73 \%$ de los productores suplementa estratégicamente con energía y/o proteína a los bovinos.
El clúster 2, integrado por el $24 \%$ de los productores de la muestra, es el grupo que incluye los establecimientos de mayor tamaño. El productor típico del clúster 2 toma una pequeña proporción de tierras en arrendamiento, $\mathrm{y}$, por lo tanto, la superficie propia promedio del grupo es levemente inferior a la superficie ganadera. En estos establecimientos, el porcentaje de engorde es sensiblemente mayor y la participación de la familia en el aporte de mano de obra es substancialmente menor, cuando se lo compara con el clúster 1. La dotación total de mano de obra es similar al primer clúster. Este grupo logra, en promedio, tres puntos porcentuales más de destete que el clúster 1 , aunque esta diferencia no es estadísticamente significativa. Este clúster se caracteriza por ser tecnológicamente superior al clúster 1 , ya que entora a edad más temprana, el servicio es de 5 meses de duración, tiene un 6\% de superficie ocupada con verdeos y el $86 \%$ de los productores suplementa con energía y/o proteína de manera estratégica a los bovinos.

Los resultados del análisis de clústers indican que al final del período analizado, convivían dos grupos de productores. Un grupo más numeroso con menor escala de producción, mayor aporte de mano de obra familiar y una tecnología ganadera menos sofisticada, junto a otro grupo menos numeroso caracterizado por una mayor escala de producción, mayor orientación al engorde de bovinos y una tecnología ganadera más avanzada. A pesar de estas diferencias, nuestros resultados indican que ambos grupos logran

Tabla 4. Valores promedio de las variables para cada grupo obtenido, prueba $\mathrm{F}$ y $\mathrm{p}$-valores para las diferencias entre grupos.

Table 4. Mean values for the variables of each cluster, $F$ test and $p$ values for the differences in means.

\begin{tabular}{|c|c|c|c|c|c|}
\hline Variables & & $\begin{array}{l}\text { Promedio } \\
\text { clúster } 1 \\
(76 \%)\end{array}$ & $\begin{array}{l}\text { Promedio } \\
\text { clúster } 2 \\
(24 \%)\end{array}$ & Prueba F & p-valor \\
\hline \multicolumn{6}{|l|}{ Estructurales } \\
\hline Sup. propia & ha & 1837 & 4590 & 24,55 & $<0,01$ \\
\hline Sup. ganadera & ha & 1803 & 4661 & 26,27 & $<0,01$ \\
\hline Exist. bovina & $\mathrm{Cab}$ & 1318 & 3381 & 24,47 & $<0,01$ \\
\hline Personal c/500 EV & Pers & 1,28 & 1,34 & 0,04 & 0,84 \\
\hline MO familiar & $\%$ & 14,37 & 4,27 & 1,93 & 0,18 \\
\hline Engorde bovino & $\%$ & 7,25 & 24,66 & 30,46 & $<0,01$ \\
\hline Carga ovina & $\%$ & 7,05 & 6,15 & 0,15 & 0,70 \\
\hline \multicolumn{6}{|l|}{ Tecnológicas } \\
\hline Edad entore & Meses & 24 & 21 & 2,64 & 0,12 \\
\hline Servicio bovino & Días & 177 & 150 & 1,24 & 0,28 \\
\hline Uso verdeos & $\%$ & 0,39 & 5,95 & 12,43 & $<0,01$ \\
\hline \multicolumn{6}{|l|}{ Productivas } \\
\hline Carga animal & $\mathrm{EV} / \mathrm{ha}$ & 0,77 & 0,72 & 0,43 & 0,52 \\
\hline Destete bovino & $\%$ & 72,99 & 76,11 & 0,72 & 0,40 \\
\hline
\end{tabular}


una productividad similar, al evaluarla a través de la carga animal y el porcentaje de destete, destacándose el grupo 1 en el porcentaje de señalada alcanzada en ovinos.

\section{CONCLUSIONES}

Este trabajo ha cuantificado y analizado los cambios ocurridos en la estructura, tecnología y productividad de establecimientos ganaderos del Centro Sur de la provincia de Corrientes en el período 2005-2015. El análisis se realizó en dos niveles, a nivel de la muestra en su conjunto e identificando a nivel de los casos individuales qué características al inicio del período se asocian con los cambios registrados al final del período de análisis. No se registran cambios de magnitud en los valores promedio de la superficie propia ni de la superficie ganadera, pero se observa un aumento en el uso de verdeos, una disminución en la duración del servicio de monta y un incremento en el uso de la suplementación energético-proteica. Estos cambios muestran una tendencia hacia la intensificación de la producción y hacia un uso más eficiente del capital hacienda.

A nivel de las superficies, se observa que los establecimientos que en 2005 tenían una mayor proporción de superficie alquilada y una mayor proporción de engorde lograron incrementar su superficie propia. Por otro lado, los que más redujeron la proporción de superficie tomada en alquiler fueron los casos con mayor participación de engorde bovino al inicio del período. Estos cambios pueden haber sido promovidos por los cambios en las relaciones entre los precios del ternero, del novillo y del maíz.

Las transformaciones registradas sugieren cambios tendientes a una intensificación de la producción. Los resultados presentados indican que los establecimientos con mayor orientación hacia la cría bovina, que al inicio del período llevaban adelante una producción más extensiva, han implementado cambios tendientes a intensificar la producción. Además, los resultados muestran que los casos que tenían menor dotación de mano de obra han incrementado su personal a lo largo del período analizado.

Otra serie de cambios está liderada por las explotaciones de mayor escala de producción. Estos establecimientos incrementaron su carga animal y el uso de verdeos, medidas de manejo que se asocian a un aumento en el porcentaje de destete. Finalmente, los establecimientos de mayor escala emplean un menor tiempo de servicio de monta y realizan el primer entore a menor edad de las vaquillonas, lo que sugiere un diferencial tecnológico favorable a este tipo de explotaciones.
El análisis de clústers caracterizó la diversidad de situaciones existentes indicando que, al final del período de análisis, podían identificarse dos grupos de explotaciones con características contrastantes. Se diferenciaban así dos grupos con distinta escala de producción y tecnología ganadera, pero a pesar de estas diferencias la carga animal y el porcentaje de destete eran similares y el porcentaje de señalada era mayor para el grupo con menor tecnología ganadera.

\section{AGRADECIMIENTOS}

Los autores agradecen especialmente a todos los productores que atentamente nos recibieron e informaron sobre sus establecimientos y el manejo de la actividad agropecuaria que realizan. Por otro lado, este trabajo ha recibido financiamiento del Proyecto de INTA, PNSEPT 1129023.

\section{LITERATURA CITADA}

Bert, F.E., G.P. Podestá, S.L. Rovere, A.N. Menéndez, M. North, E. Tatara, et al. 2011. An agent based model to simulate structural and land use changes in agricultural systems of the argentine pampas. Ecol. Modell. 222(19):3486-3499.

Calvi, M. 2008. Perfil tecnológico de la producción primaria. Centro Regional Corrientes, resultados en carne vacuna. 9 p. Artículo, INTA Área Estratégica de Economía y Sociología. Disponible en: https://inta.gob. ar/sites/default/files/script-tmp-perfil_ tecnologico_corrientes.pdf (Consulta 18 septiembre 2018).

Calvi, M. 2009. Análisis y evaluación de estrategias tecnológicas y productivas de los sistemas de producción ganaderos predominantes en el Centrosur de Corrientes. 168 p. Tesis Magister en Agroeconomía. Unidad Integrada Balcarce, Universidad Nacional de Mar del Plata - INTA, Balcarce, Argentina.

Cetrá, B., L. Rivero, y M. Pereira. 2002. Parásitos. Resistencia a los antiparasitarios en rumiantes. 3 p. INTA EEA Mercedes, Noticias y Comentarios № 367. ISSN № 0327-3059. Mercedes (Corrientes), Argentina.

Giancola, S., S. Calvo, D. Sampedro, A. Marastoni, V. Ponce, S. Digiano, et al. 2013. Causas que afectan la adopción de tecnología en la ganadería bovina para carne de la provincia de Corrientes. Enfoque cualitativo. 62 p. Ed. INTA, Serie Estudios Socioeconómicos de la Adopción de Tecnología N² 2. ISSN N²3141727. Buenos Aires, Argentina. 
Greenacre, M. 2017. Correspondence analysis in practice. 326 p. $3^{\text {rd }}$. ed. ISBN № 9781498731782. Chapman \& Hall/CRC, New York, USA.

Gonzalez, M.D.C. (Coord.). 2005. Productores familiares pampeanos: hacia la comprensión de similitudes y diferenciaciones zonales. Astralib, Cooperativa Editora. Buenos Aires, Argentina.

Paruelo, J.M., J.P. Guerschman, y S.R. Verón. 2005. Expansión agrícola y cambios en el uso del suelo. Revista Ciencia Hoy Vol. 15 № 87, p. 14-23. ISSN № 1666-5171.

Pereira, D., D. Formoso, H. Deschenaux, M.L. Del Pino, D. Castells y J. Piaggio. 2014. Pastoreo mixto: estrategia para alcanzar beneficios sostenibles. p 20-23. Revista Ovinos SUL, Edición Mayo 2014.

Pueyo, J., R. Ordenavía, R. Pizzio, J. Fernández, C. Benítez, y O. Royo Pallares. 1993. Evaluación de la vegetación y de la producción animal con dos relaciones lanar/vacuno sobre campo natural en el sur de la provincia de Corrientes. p. 139-142. Resumen Anual, INTA EEA Mercedes. Mercedes (Corrientes), Argentina.

Rada, N.E. and K.O. Fuglie. 2018. New perspectives on farm size and productivity. Food Policy. Available at https://www. researchgate.net/publication/323997279_ New_perspectives_on_farm_size_and_ productivity (Accessed 24 March 2018). doi: 10.1016/j.foodpol.2018.03.015.
Rearte, D. 2010. Situación actual y prospectiva de la producción de carne vacuna. 26 p. Informe INTA. Programa Nacional Carnes. Disponible en https://inta.gob.ar/documentos/situacionactual-y-prospectiva-de-la-produccion-decarne-vacuna (Consulta 18 septiembre 2018).

Ricard, M.F., E.F. Viglizzo, and G. Podestá. 2015. Comparison of adaptative strategies to climate variability in rural areas of Argentine Chaco and US Southern Plains during the last century. Journal of Arid Environments 123:68-80. https://doi.org/10.1016/j. jaridenv.2014.10.009

SENASA. 2016. Existencia bovina y establecimientos agropecuarios, año 2016. Disponible en: http://www.senasa.gob.ar (Consulta 7 julio 2016).

Velasco Fuenmayor, J., L. Ortega Soto, E. Sánchez Camarillo, y F. Urdaneta. 2009. Factores que influyen sobre el nivel tecnológico presente en las fincas ganaderas de doble propósito localizadas en el estado Zulia, Venezuela. Revista Científica, Fac. Ciencias Veterinarias Univ. del Zulia (LUZ) 19(2):187-195. ISSN 0 798-2259. 